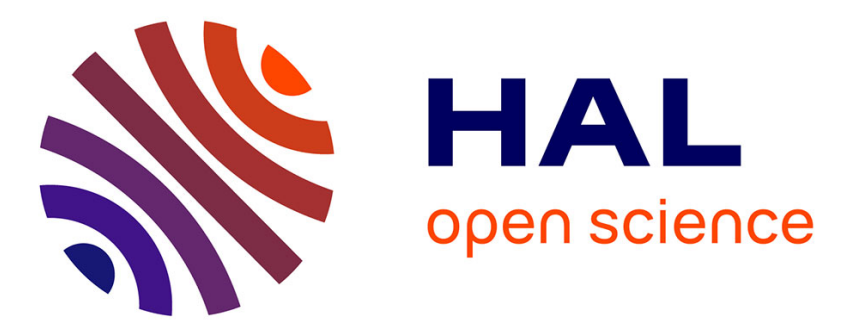

\title{
Lagrangian trajectory model for turbulent swirling flow in an annular cell: comparison with residence time distribution measurements
}

\author{
Jeremy Pruvost, J. Legrand, P. Legentilhomme, A. Muller-Feuga
}

\section{To cite this version:}

Jeremy Pruvost, J. Legrand, P. Legentilhomme, A. Muller-Feuga. Lagrangian trajectory model for turbulent swirling flow in an annular cell: comparison with residence time distribution measurements. Chemical Engineering Science, 2002, 57 (7), pp.1205-1215. 10.1016/S0009-2509(02)00009-X . hal02534005

\section{HAL Id: hal-02534005 \\ https://hal.science/hal-02534005}

Submitted on 17 Apr 2020

HAL is a multi-disciplinary open access archive for the deposit and dissemination of scientific research documents, whether they are published or not. The documents may come from teaching and research institutions in France or abroad, or from public or private research centers.
L'archive ouverte pluridisciplinaire HAL, est destinée au dépôt et à la diffusion de documents scientifiques de niveau recherche, publiés ou non, émanant des établissements d'enseignement et de recherche français ou étrangers, des laboratoires publics ou privés. 


\title{
Lagrangian trajectory model for turbulent swirling flow in an annular cell: comparison with residence time distribution measurements
}

\author{
J. Pruvost ${ }^{\mathrm{a}}$, J. Legrand ${ }^{\mathrm{a}, *}$, P. Legentilhomme ${ }^{\mathrm{a}}$, A. Muller-Feuga ${ }^{\mathrm{b}}$ \\ ${ }^{a}$ GEPEA-UMR.MA 100, University of Nantes, CRTT-IUT, BP 406, F-44602 Saint-Nazaire Cedex, France \\ bPA Laboratory, IFREMER, Rue de l'Ile d'Yeu, BP21105, F-44311 Nantes Cedex 3, France
}

\begin{abstract}
Knowledge of the flow field can be very useful for reactor design and optimization. Data concerning the displacement and trajectories of reactive elements are of primary interest for a better understanding of process running. Results can be deduced from computational fluid dynamics or experimental measurements. However, numerical simulation is difficult to achieve for complex flow such as swirling decaying flow, and trajectories need to be calculated on the basis of velocity field measurements. This problem can be simplified by using a Lagrangian formulation, in which case the only major difficulty is to express the influence of turbulence on calculated trajectories. Velocity fluctuations can be considered as pure random functions or related to turbulence correlations. These two methods were used to calculate trajectories of elementary fluid particles in a swirling decaying flow on the basis of hydrodynamic characteristics obtained by particle image velocimetry (PIV) studies. Trajectory dispersion was then compared with experimental results obtained by PIV measurements of the instantaneous flow field. This comparison shows the great dependence of velocity fluctuation (and thus of trajectories) on spatial correlations. Finally, the correct mathematical formulation of velocity fluctuation was checked by using the trajectory calculation algorithm to determine residence time distribution (RTD). A comparison with experimental RTD confirmed the efficiency of the method for determining trajectories in swirling decaying flow.
\end{abstract}

Keywords: Hydrodynamics; Trajectory; Mixing; Turbulence; Swirling flow; Transport processes

\section{Introduction}

Trajectories can provide useful information about chemical processes, but are difficult to measure accurately. Visualization techniques such as the injection of a coloring agent or a particle tracer (e.g. glass, polystyrene or fog) only provide a global estimation of trajectories. Another solution is to use computational fluid dynamics (CFD) to model the flow under study. The velocity field can then be determined, making it easy to deduce elementary fluid trajectories (Farias Neto, Legentilhomme, \& Legrand, 1998). However, when flow exhibits complex behavior, modeling becomes very difficult, especially in a turbulent flow regime, so that experimental investigation is often reauired.
Recent experimental velocity measurement techniques, such as laser Doppler anemometry (LDA) and hot-wire or particle image velocimetry (PIV), provide an accurate representation of the main hydrodynamic characteristics (e.g. instantaneous components of the velocity field). Trajectories of elementary particles can then be deduced by integrating the velocity field. If the flow field is complex, no analytical solution is available and numerical resolution is necessary. For this purpose, a Lagrangian formulation is often applied in CFD (Berlemont, Desjonqueres, \& Gouesbet, 1990; Chen \& Pereira, 1998; Domgin, Huilier, Burnage, \& Gardin, 1997). Trajectories are then determined step by step by calculating the successive positions of the fluid element $P$ by

$P(t+\Delta t)=P(t)+u \Delta t$,

where $\Delta t$ is a time step to be determined and $u$ is the instantaneous velocity. 


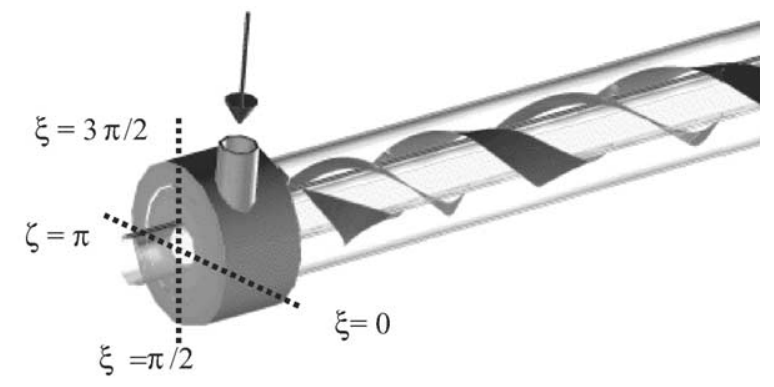

Fig. 1. Schematic representation of the tangential inlet and swirling decaying flow.

If the Reynolds' decomposition of instantaneous velocity is used in Eq. (1), the path of an elementary fluid particle can be deduced by

$P(t+\Delta t)=P(t)+\left(U+U^{\prime}\right) \Delta t$,

where $U$ is the mean value of velocity and $U^{\prime}$ the fluctuating one.

Mean statistical characteristics of flow, such as mean velocity components, turbulence intensities or velocity correlations, can be measured experimentally. If mean velocity distribution is known in Eq. (2), the remaining difficulty is to determine the fluctuating velocity $U^{\prime}$ due to turbulence. This can be solved by mathematical modeling of the fluctuating velocity (Berlemont et al., 1990). Two commonly employed methods were used here and compared for swirling decaying annular flow.

This type of flow is induced in an annular geometry, using a tangential inlet as a simple design for this purpose (Gupta, Lilley, \& Syred, 1984). A schematic representation of the geometry is shown in Fig. 1. This flow, which exhibits three-dimensional motion and increasing turbulence intensities as compared to that obtained in purely axial flow, is an efficient means of enhancing heat or mass transfer phenomena (Legentilhomme \& Legrand, 1990, 1991; Legentilhomme, Aouabed, \& Legrand, 1993). Swirl motion is created at the inlet of the device and then decays freely along the flow path, displaying very disturbed and complex flow behavior (Aouabed, Legentilhomme, Nouar, \& Legrand, 1994; Aouabed, Legentilhomme, \& Legrand, 1995; Legrand, Aouabed, Legentilhomme, Lefèbvre, \& Huet, 1997). However, numerical simulation of this flow is difficult to achieve. An easy way to investigate this flow is to use the PIV measurement technique to provide the main hydrodynamic characteristics. A previous study showed how the turbulence length scale and the three components of mean velocity and turbulence intensity can be obtained with this technique (Pruvost, Legrand, Legentilhomme, \& Doubliez, 2000). In the present study, experimental PIV measurements were used to determine trajectories with Eq. (2). However, swirling flow is not axisymmetrical, which means that a three-dimensional calculation method is also needed. For validation, residence time distribution (RTD) was determined experimentally and compared with that simulated by the trajectory calculation algorithm.

\section{Experimental setup}

The hydraulic test rig used for the experimental investigation, which was previously described in Pruvost et al. (2000), consists of an annular test section (Fig. 1) composed of two concentric Plexiglas tubes (a total length of $1.5 \mathrm{~m}$ ). The internal radius of the outer cylinder $R_{o}$ is $50 \mathrm{~mm}$, and the outer radius of the inner tube $R_{i}$ is $20 \mathrm{~mm}$. The diameter of the tangential inlet is $30 \mathrm{~mm}$, equal to the annular gap width $e$ corresponding to pure swirling flow according to Legentilhomme and Legrand (1991). The outlet of the annulus is axial in order to avoid flow disturbance. For all experimental measurements, the water flow rate is $Q=1.27 \mathrm{~m}^{3} / \mathrm{h}$, corresponding to a mean axial velocity in the annulus, $\bar{U}$, of $5 \mathrm{~cm} / \mathrm{s}$ and a Reynolds number $R e$ of 3000 , where $R e$ is calculated by

$\operatorname{Re}=\frac{2 e \bar{U}}{v}$.

All hydrodynamic characteristics described in this study were obtained by the PIV measurement technique. The PIV setup and the investigation method are described in Pruvost et al. (2000). However, PIV cannot provide data concerning the effect of the flow field on the entire geometry. To calculate trajectories with a Lagrangian formulation, each hydrodynamic parameter needs to be determined for successive locations of the elementary fluid particle. On the basis of experimental PIV results (Pruvost, Legrand, \& Legentilhomme, 2001), a neural network method employing radial basis functions (RBF) was used to obtain continuous expression of each of these characteristics. This technique allows hydrodynamic properties to be determined, such as the mean velocity field and turbulence intensities, on the entire geometry of the test set.

The mean velocities and turbulence intensities calculated from PIV instantaneous results were defined by

$$
\begin{aligned}
U_{x} & =\frac{1}{N} \sum_{i=1}^{N} u_{x_{i}}, \quad U_{r}=\frac{1}{N} \sum_{i=1}^{N} u_{r_{i}}, \quad U_{\zeta}=\frac{1}{N} \sum_{i=1}^{N} u_{\zeta_{i}}, \\
T_{x} & =\frac{\sqrt{(1 / N) \sum_{i=1}^{N}\left(u_{x_{i}}-U_{x}\right)^{2}}}{\bar{U}}, \\
T_{r} & =\frac{\sqrt{(1 / N) \sum_{i=1}^{N}\left(u_{r_{i}}-U_{r}\right)^{2}}}{\bar{U}}, \\
T_{\zeta} & =\frac{\sqrt{(1 / N) \sum_{i=1}^{N}\left(u_{\zeta_{i}}-U_{\zeta}\right)^{2}}}{\bar{U}} .
\end{aligned}
$$

To achieve stable results, 200 PIV acquisitions were needed for mean values, and 1000 for turbulence intensities. An example of velocity distributions is shown in Fig. 2 for the angular position $\xi=0$. 


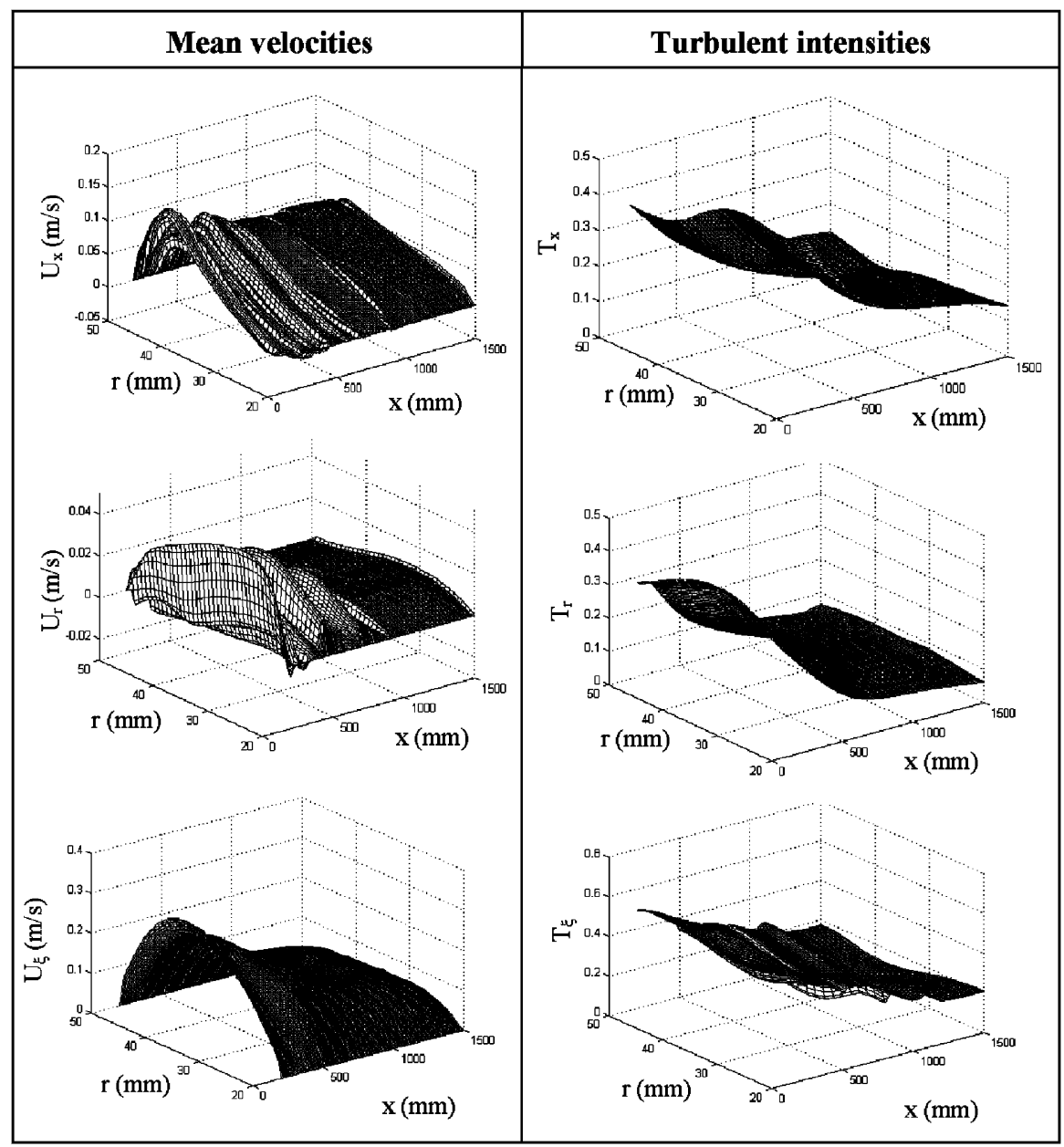

Fig. 2. Mean velocities and turbulence intensity distributions obtained using PIV for $\xi=0$.

\section{Modeling of velocity fluctuations in a swirling flow}

\subsection{Mathematical formulation}

Trajectory calculation is often used in two-phase flow modeling (Berlemont et al., 1990; Berlemont, Chang, \& Gouesbet, 1998; Chen \& Pereira, 1996; Domgin et al., 1997; Ormancey \& Martinon, 1984). According to these studies, fluctuating velocity needs to be considered carefully to achieve accurate results. Some mathematical formulations of this time-dependent parameter are available (Berlemont et al., 1990; Ormancey \& Martinon, 1984), and two methods were employed in the present study. The first and simpler one used a random function of Gaussian type with its root mean square linked to turbulence intensity (method $\mathrm{A})$ :

$U^{\prime}(P)=q(P)$,

where $q(P)$ is a Gaussian distributed random function determined for the location $P$.

This formulation can be sufficient, even though it is not fully representative of the real turbulence- field. For anisotropic three-dimensional turbulence, the expression is

$$
\begin{aligned}
& U_{x}^{\prime}(x, r, \xi)=q_{x}\left(m_{x}, \tau_{x}\right)=q_{x}\left(0, \bar{U} T_{x}(x, r, \xi)\right), \\
& U_{r}^{\prime}(x, r, \xi)=q_{r}\left(m_{r}, \tau_{r}\right)=q_{r}\left(0, \bar{U} T_{r}(x, r, \xi)\right), \\
& U_{\zeta}^{\prime}(x, r, \xi)=q_{\zeta}\left(m_{\zeta}, \tau_{\zeta}\right)=q_{\zeta}\left(0, \bar{U} T_{\xi}(x, r, \xi)\right),
\end{aligned}
$$

where $m$ and $\tau$ are, respectively, the mean and the root mean square of the Gaussian random function calculated for each velocity component.

However, the approximation of fluctuating velocities by a pure random phenomenon leads to a loss of information, especially for turbulence correlations that cannot be neglected in duct flows (Hinze, 1959). Thus, a second method was used to take turbulence correlations into account when calculating fluctuation velocity (method B). This formulation corresponds to the weighted sum of a random term plus another term for correlation expression (Berlemont et al., 1990):

$U^{\prime}(P+\Delta P)=c_{\mathrm{cor}} U^{\prime}(P)+c_{\mathrm{rand}} q(P)$, 
where $c_{\text {cor }}$ and $c_{\text {rand }}$ are the weighting parameters to be determined, linked, respectively, to the correlation and random terms.

\subsection{Spatial correlations of fluctuating velocities}

Expressions of parameters $c_{\text {cor }}$ and $c_{\text {rand }}$ depend on the type of flow under study, and especially on the turbulence correlation obtained. As shown in a previous study (Pruvost et al., 2000), the PIV technique is quite suitable for measurement of spatial correlations. Thus, a preliminary experimental investigation was carried out.

Spatial correlation values can be obtained using PIV for both axial and radial velocity components along the axial and radial coordinates. Spatial correlations are defined by

$f_{x, r, \xi}\left(l_{x}\right)=\frac{\left\langle U_{x}^{\prime}(x, r, \xi) U_{x}^{\prime}\left(x+l_{x}, r, \xi\right)\right\rangle}{\left\langle U_{x}^{\prime}(x, r, \xi)^{2}\right\rangle}$,

$f_{x, r, \xi}\left(l_{r}\right)=\frac{\left\langle U_{x}^{\prime}(x, r, \xi) U_{x}^{\prime}\left(x, r+l_{r}, \xi\right)\right\rangle}{\left\langle U_{x}^{\prime}(x, r, \xi)^{2}\right\rangle}$,

$g_{x, r, \xi}\left(l_{x}\right)=\frac{\left\langle U_{r}^{\prime}(x, r, \xi) U_{r}^{\prime}\left(x+l_{x}, r, \xi\right)\right\rangle}{\left\langle U_{r}^{\prime}(x, r, \xi)^{2}\right\rangle}$,

$g_{x, r, \xi}\left(l_{r}\right)=\frac{\left\langle U_{r}^{\prime}(x, r, \xi) U_{r}^{\prime}\left(x, r+l_{r}, \xi\right)\right\rangle}{\left\langle U_{r}^{\prime}(x, r, \xi)^{2}\right\rangle}$,

where \langle\rangle represents spatial averaging, $f_{x, r, \xi}\left(l_{x}\right)$ is the axial correlation of the axial velocity component, $g_{x, r, \xi}\left(l_{x}\right)$ the axial correlation of the radial velocity component, $f_{x, r, \xi}\left(l_{r}\right)$ the radial correlation of the axial velocity component, and $g_{x, r, \xi}\left(l_{r}\right)$ the radial correlation of the radial velocity component.

To decrease the amount of stored data, simplified functions can be used to fit spatial correlations (Berlemont et al., 1990; Hinze, 1959). Fig. 3 shows an example of experimental correlation calculated by using PIV and two kinds of commonly used functions (Hinze, 1959). Gaussian type functions appeared to be more suitable. Such functions depend only on one fitting parameter, known as the integral

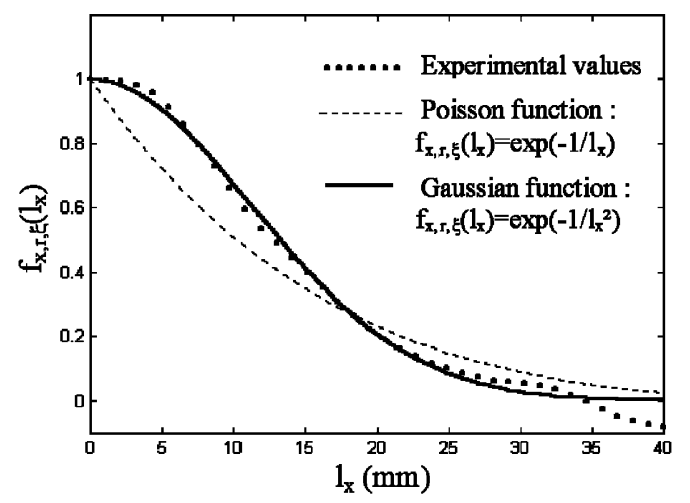

Fig. 3. Example of spatial correlation obtained using PIV and fitting curves. length scale $L$, defined by

$F(l)=\exp \left(-\left(\frac{l}{L}\right)^{2}\right)$.

The integral length scale was calculated for each spatial correlation: $L_{f x}$ for the axial correlation of the axial velocity component, $L_{g x}$ for the axial correlation of the radial velocity component, $L_{f r}$ for the radial correlation of the axial velocity component, and $L_{g r}$ for the radial correlation of the radial velocity component.

The length scales obtained along the axial coordinate are shown in Fig. 4. Because of the small width of the annular gap, correlations in the radial direction were measured for only one radial position. The results indicate a radial symmetry of velocity fluctuations when correlations are calculated along the half size of the annular gap width. Thus, radial correlation can be fully determined if measurements are made in the middle of the annular gap $(r=25 \mathrm{~mm})$, both for increasing $\left(r_{\text {sup }}\right)$ and decreasing $\left(r_{\text {inf }}\right)$ radial values. The results calculated along the radial coordinate are also shown in Fig. 4.

If Eq. (13) is used to represent the spatial correlation in Eq. (8), axial and radial fluctuating velocities can be determined (Berlemont et al., 1990; Ormancey \& Martinon, 1984) by

$U^{\prime}(P+\Delta P)=c_{\text {cor }} U^{\prime}(P)+c_{\text {rand }} q(P)$

with

$c_{\text {cor }}=\exp \left(-\left(\frac{\Delta P}{L}\right)^{2}\right) \quad$ and

$c_{\text {rand }}=\sqrt{1-\exp \left(-\left(\frac{\Delta P}{L}\right)^{2}\right)}$.

The three-dimensional calculation of trajectories allows integral length scale to be determined on the entire geometry. However, the results given in Fig. 4 indicate that these values can be approximated as axisymmetrical (nearly the same results are obtained for each investigated angular position). To simplify the numerical determination of trajectories and decrease computation time, the distributions indicated in Fig. 4 were averaged over the circumferential coordinate. Thus, the integral length scale parameters in method B are only a function of axial and radial coordinates.

Finally, it should be noted that this method does not apply to circumferential velocity, because the PIV technique cannot provide measurement of linked spatial correlations. It was chosen to consider an additional hypothesis, i.e. the conservation of turbulent energy $K$ when velocity fluctuations are determined. After axial and radial fluctuating velocities were calculated, the following equation was used:

$U_{\zeta}^{\prime}=\sqrt{\left|\bar{U}^{2} K-U_{x}^{\prime 2}-U_{r}^{\prime 2}\right|}$. 


\subsection{Trajectory calculation algorithms}

Trajectories can be calculated using method A or B. Method A is very simple. Successive positions of the fluid element are calculated using Eq. (2), and velocity fluctuation is deduced for each step according to Eq. (7). Hydrodynamic characteristics are determined for each position using the results of a reconstruction method based on a neural network algorithm (Pruvost et al., 2001), which can be expressed by the following algorithm:

\section{Definition of the starting location \\ While the fluid particle stays in the geometry a-Determination of hydrodynamic characteristics for the current position using neural network results (mean velocities, turbulence intensities) $b$-Determination of fluctuating velocities using Eq. (7) End $c$-Determination of the next position using Eq. (2)}

Unlike method A, method B involves a further hypothesis, as explained by Ormancey and Martinon (1984). In Eq. (14), the integral length scale is determined for the starting position of the fluid particle. However, when the particle moves away, the influence of the spatial correlation term decreases, until velocity fluctuation becomes only a random function. This can be taken into account by introducing an arbitrary value that gives the maximum length covered by the fluid particle before the current position is redefined as the new initial point for the spatial correlation. Different values can be found, ranging between $L / 2$ and $3 L / 2$ (Ormancey \& Martinon, 1984; Berlemont et al., 1990). Because these data can only be defined arbitrarily, the $L / 2$ value was chosen to provide accurate consideration of the spatial correlation by frequent redetermination of the integral length scale. Thus, the final algorithm for method B is

1-Definition of the starting location

While the fluid particle stays in the geometry

2-Determination of integral length scales for the current position

When the distance covered is less than $L / 2$ $2 a$-Determination of hydrodynamic characteristics for the current position using neural network results (mean velocities, turbulence intensities)

$2 b$-Determination of fluctuating velocities using Eqs. (14) and (15)

$2 c$-Determination of the next position using Eq. (2)

End

End

\subsection{Experimental validation}

To compare methods A and B, experimental trajectories were approximated using PIV measurements to provide an instantaneous velocity field in a designated area. Therefore, the measured velocities are the exact representation of velocity $u$ in Eq. (1), which can be determined with method A or B. A set of experimental trajectories can thus be deduced from the measured velocity field, even though trajectory dispersion is not fully representative of reality. There are two reasons why the dispersion calculated from PIV results can be regarded only as an approximation:

- First, the motion involved is three-dimensional, but only two-dimensional instantaneous velocity fields were measured. However, the objective was to compare methods $\mathrm{A}$ and $\mathrm{B}$ with respect to the simulation of fluctuating velocities. Thus, this preliminary study can be reduced to a two-dimensional one and the two methods will be compared for only two of the velocity components.

- Secondly, PIV gives instantaneous velocity simultaneously at different locations (the acquisition area). However, in Lagrangian formulation, the velocity field changes for each successive step $\Delta t$. The measured velocity field is thus not truly valid for determination of real trajectories.

Despite these two drawbacks, our results show that this preliminary study is sufficient to approximate trajectory dispersion due to turbulence and to compare methods $\mathrm{A}$ and B. To estimate real dispersion, a set of 200 instantaneous acquisitions was made using PIV. A starting location was determined, and the trajectories were deduced according to Eq. (1). The time step $\Delta t$ was set at $0.01 \mathrm{~s}$ (see Sections 4.1). Four velocity field examples are shown in Fig. 5, together with the corresponding trajectories. The trajectory dispersion obtained using the overall data set is indicated in Fig. 6. The effect of turbulence on dispersion is emphasized.

Some hydrodynamic characteristics were required to compare methods A and B. The 200 velocity fields were averaged beforehand to determine the mean velocity field and turbulence intensity distributions (Fig. 7). They were then used to calculate 200 trajectories using fluctuating velocities determined with method A [Eq. (6)] and method B [Eqs. (14) and (15)]. The resulting trajectory dispersions are shown in Fig. 8 .

A comparison of Figs. 6 and 8a shows that method A underestimates the influence of turbulence. Calculated trajectories are similar, despite the estimation of real dispersion. Method B appears to be more suitable for representing fluctuating velocity accurately (Fig. 8b). Thus, turbulence spatial correlations cannot be neglected in swirling decaying flow, because the eddies that develop appear to be spatially structured. Some eddies can be seen on the instantaneous PIV acquisitions shown in Fig. 5. The introduction of the length scale of turbulence into Eq. (14) takes into account the effect of eddies on trajectories. Thus, method B was retained for the calculation of three-dimensional trajectories in swirling flow. 

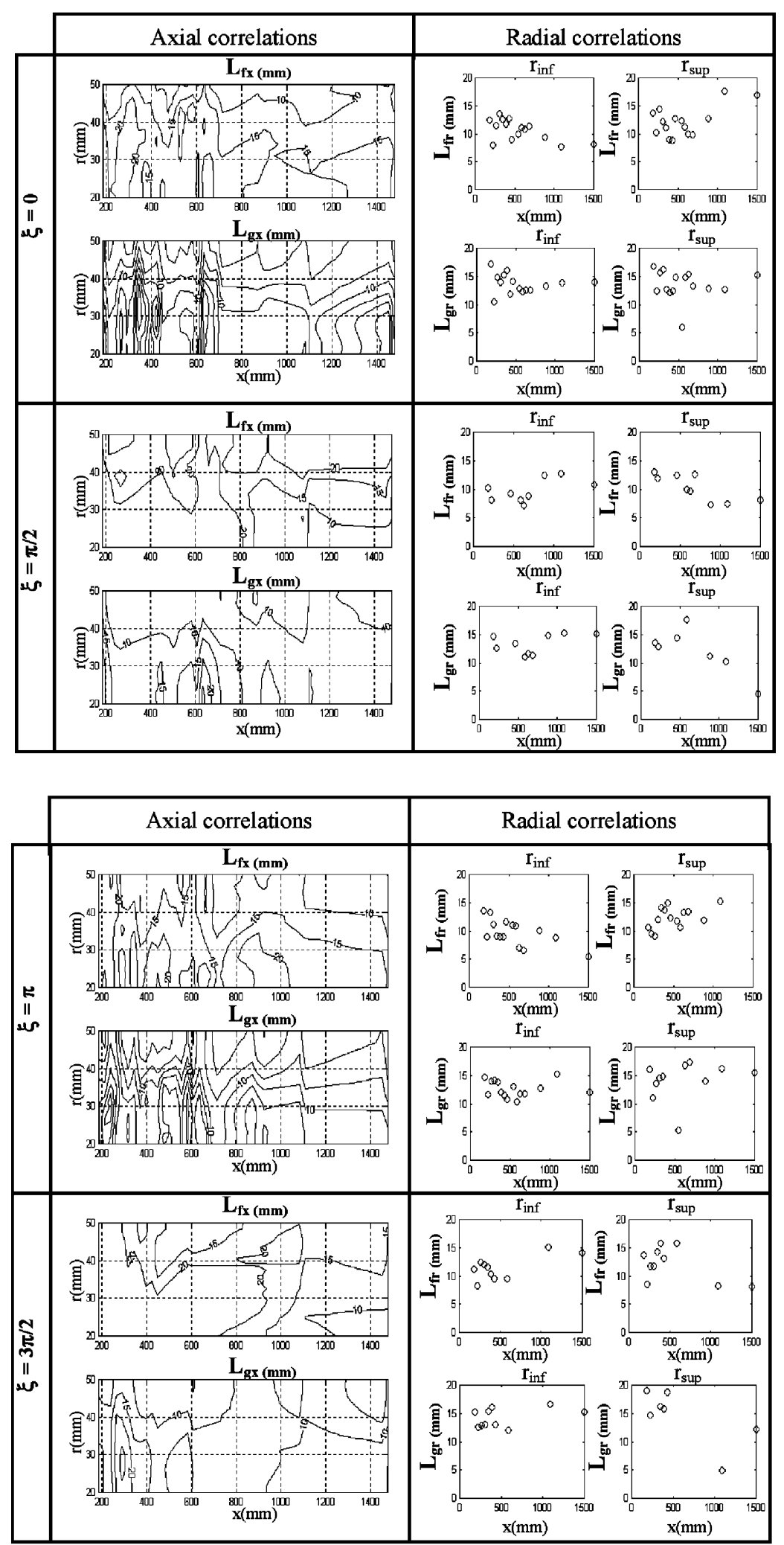

Fig. 4. Distributions of integral length scales in the axial and radial directions. 

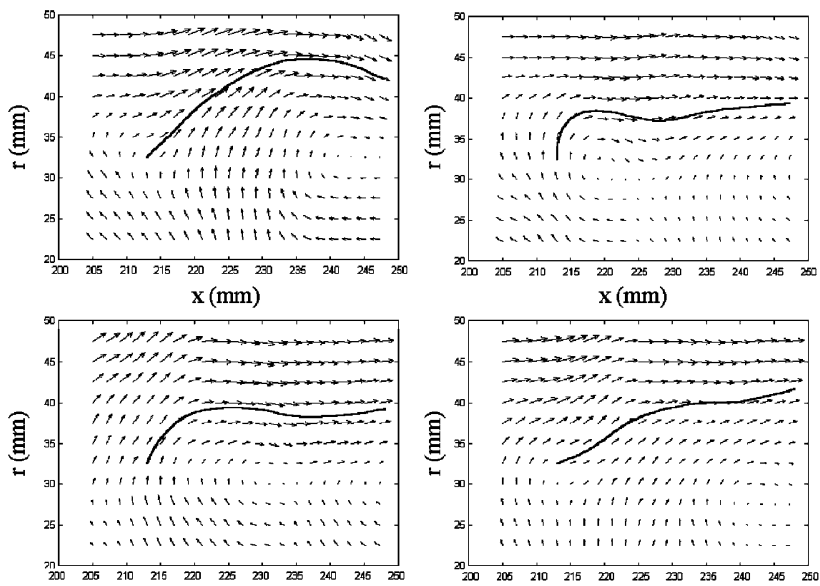

$\mathbf{x}(\mathrm{mm})$

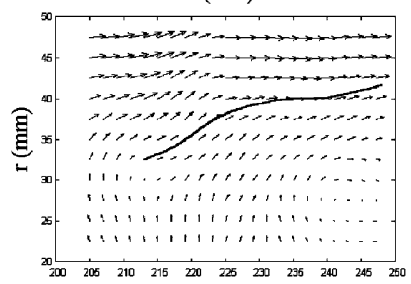

$\mathbf{x}(\mathrm{mm})$

Fig. 5. Examples of instantaneous velocity fields measured by PIV and corresponding trajectories.

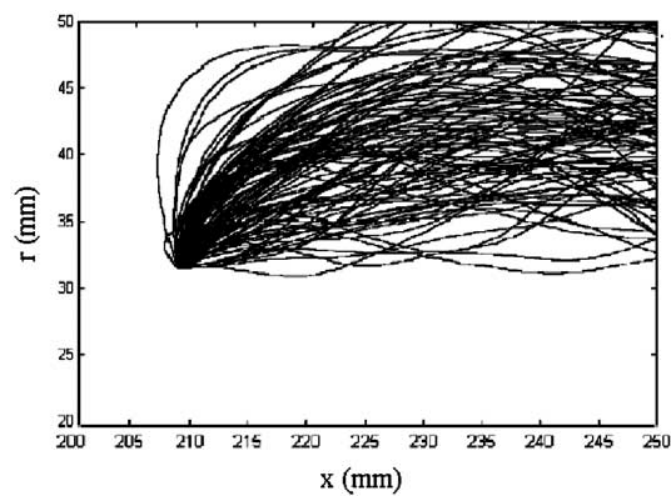

Fig. 6. Dispersion of 200 trajectories estimated experimentally.

\section{Trajectory calculation}

\subsection{Trajectory algorithm results}

In the Lagrangian formulation of Eq. (1), the time step $\Delta t$ needs to be specified. This value was determined by studying the influence of this parameter on the mean residence time of the fluid element in the cell. A set of trajectories was determined with method $\mathrm{B}$, and residence time values were averaged. The size of the set was chosen to obtain stable values. As the results in Fig. 9 indicate that trajectories are not dependent on the time step when a minimal value of $0.01 \mathrm{~s}$ is chosen, this value was retained.

Two examples of trajectories are shown in Fig. 10. The rotating motion around the inner cylinder can easily be seen on the three-dimensional representations of Figs. 10a and b. A decrease of this motion in axial position can also be observed, especially in Fig. 10b: the helicoidal pitch of trajectories increases at some distance from the inlet. These results are concordant with visualization studies using a dot-paint method (Aouabed et al., 1994, 1995). Plane projections of trajectories (Fig. 10) show the complex behavior of the flow. Elementary particle displacements appear to be very disturbed. Another important characteristic already noted by Aouabed et al. (1994) is shown in Fig. 10b, i.e. flow reversal appears near the inner cylinder for axial positions close to the inlet. Reverse flow disappears away from the inlet. Moreover, swirling flow involves not only a rotating motion, but also induces a displacement in radial direction due to the occurrence of large eddies. This accounts for the efficiency of swirling flow, which appears to create a three-dimensional motion in which mixing conditions are highly improved (Legentilhomme et al., 1993; Legentilhomme \& Legrand, 1990). The trajectory calculation algorithm appears to be a useful tool for understanding the effect of flow on trajectories and thus on the course of the fluid particle in this type of device.

\subsection{Experimental validation of the trajectory calculation algorithm}

Trajectory calculation was validated by comparing numerical results with those for experimental determination of RTD. A conductance measurement method was employed, using a two-measurement probe technique (Legentilhomme \& Legrand, 1995). Inlet and outlet probes were set as shown in Fig. 11, and an $\mathrm{NaCl}$ tracer solution was injected before the inlet probe. Each probe was connected to a conductimeter giving a signal proportional to the $\mathrm{NaCl}$ concentration. The experimental results are shown in Fig. 12.
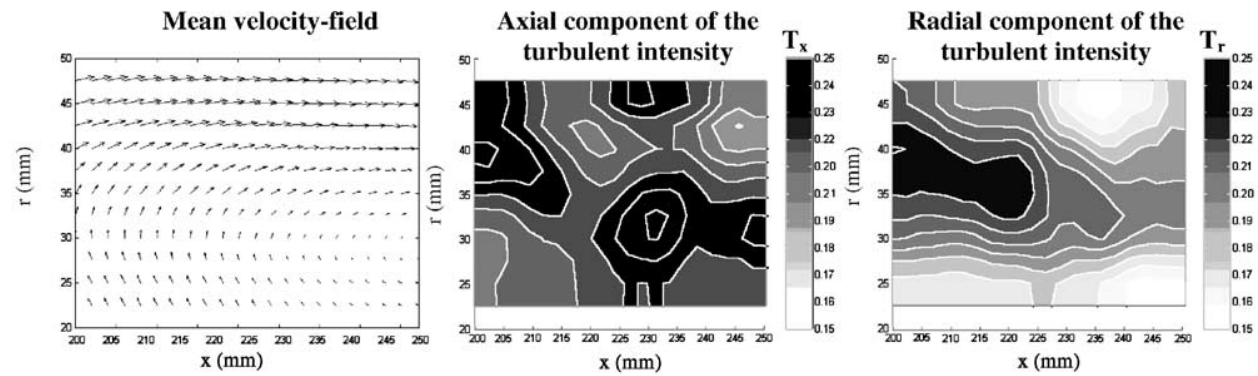

Fig. 7. Hydrodynamic parameter distributions deduced from experimental measurements of the 200 instantaneous velocity fields. 

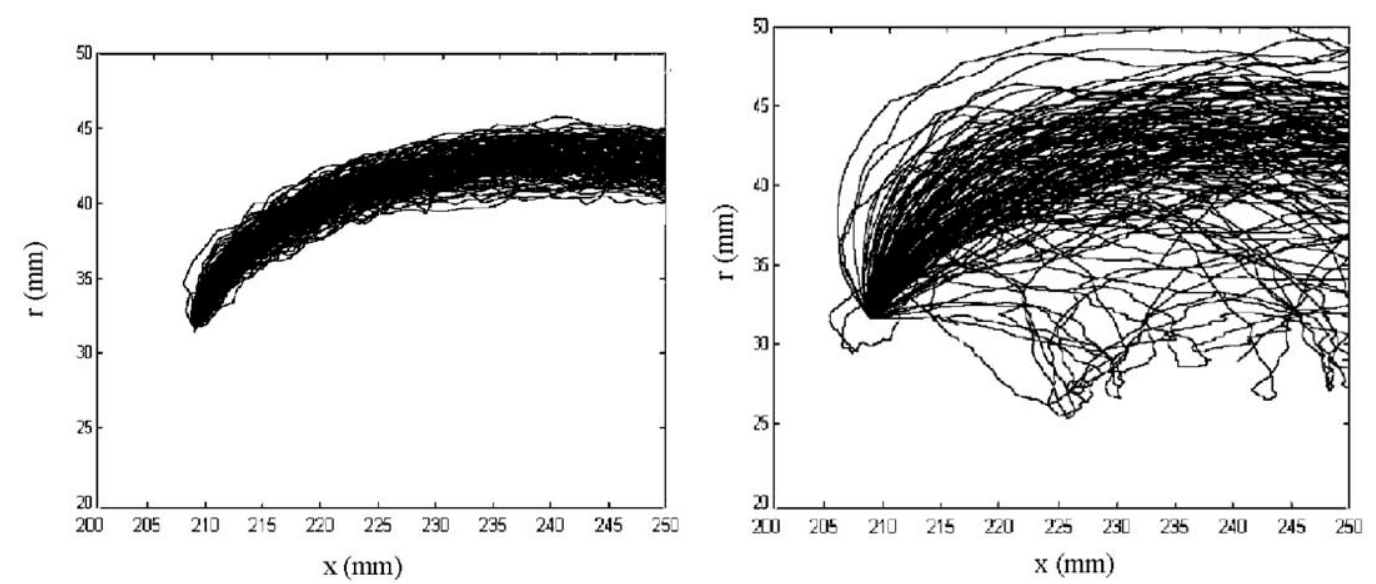

Fig. 8. Trajectory estimation using the two methods for calculating fluctuating velocities: (a) method A; (b) method B.

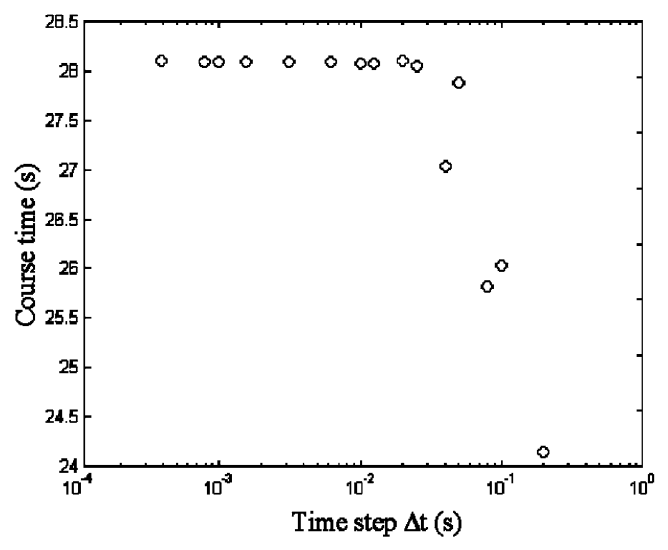

Fig. 9. Influence of the time step on the residence time calculation.

To determine RTD using the trajectory calculation algorithm, a set of 15,000 trajectories was calculated, with an initial starting point located in the inlet of the annulus. The time needed for each elementary fluid particle to reach the outlet gave RTD. However, the inlet signal was not a perfect Dirac type impulsion (Fig. 12). To obtain an accurate representation of real injection conditions, the low dispersion of the tracer at the inlet probe was simulated by dividing inlet distribution into time intervals (Fig. 13). The set of calculated trajectories was then randomly re-allocated according to divided inlet distribution and resulting calculated outlet time distribution (Fig. 14).

RTD determined with the trajectory calculation algorithm was similar to that of experimental conditions, which indicates that the algorithm is able to represent complex hydrodynamic particle dispersion in turbulent swirling flow. Fig. 14 indicates that some trajectories produced by the algorithm have a residence time of more than $60 \mathrm{~s}$. This occurs when some elementary fluid particles remain successively in recirculation areas of the swirling flow and near walls (Fig. 10b). An elementary fluid particle can remain for a long time at this location, increasing the resulting residence time. However, only $5 \%$ of trajectories have a residence time longer than $60 \mathrm{~s}$, and only $1 \%$ greater than $80 \mathrm{~s}$. Moreover, the conductance measurement method is not sensitive enough to allow determination of very small tracer amounts in solution. Thus, observation of long residence time is difficult in experimental conditions. On the whole, the trajectory calculation algorithm can be considered to be accurate in comparison with experimental results.

\section{Conclusion}

A Lagrangian method was used to calculate trajectories of elementary fluid particles in a swirling decaying flow. Experimental trajectories estimated by PIV showed the development of large eddies preventing the approximation of fluctuation velocities by a pure random phenomenon. This characteristic of the complex behavior of swirling flow was simulated by considering the integral length scale of turbulence, as measured by PIV, for the determination of fluctuating velocities. The Lagrangian model was verified by comparing the results with experimental measurement of RTD, which showed the efficiency of the method retained.

This technique allows calculation of the trajectories of elementary fluid particles from experimental results measured by PIV. The three-dimensional behavior of swirling decaying flow was determined, as well as such essential characteristics as the decay of the rotating motion created around the inner cylinder by the tangential inlet, flow reversal, and particle displacement along the radial coordinate. These characteristics are very difficult to measure when flow is complex, especially when the three-dimensional motion created by the tangential inlet prevents direct determination of trajectories. Thus, the model developed in this study appears to be useful for optimizing processes involving swirling decaying flows. The hydrodynamics involved is difficult to understand and characterize, even though these processes appear 

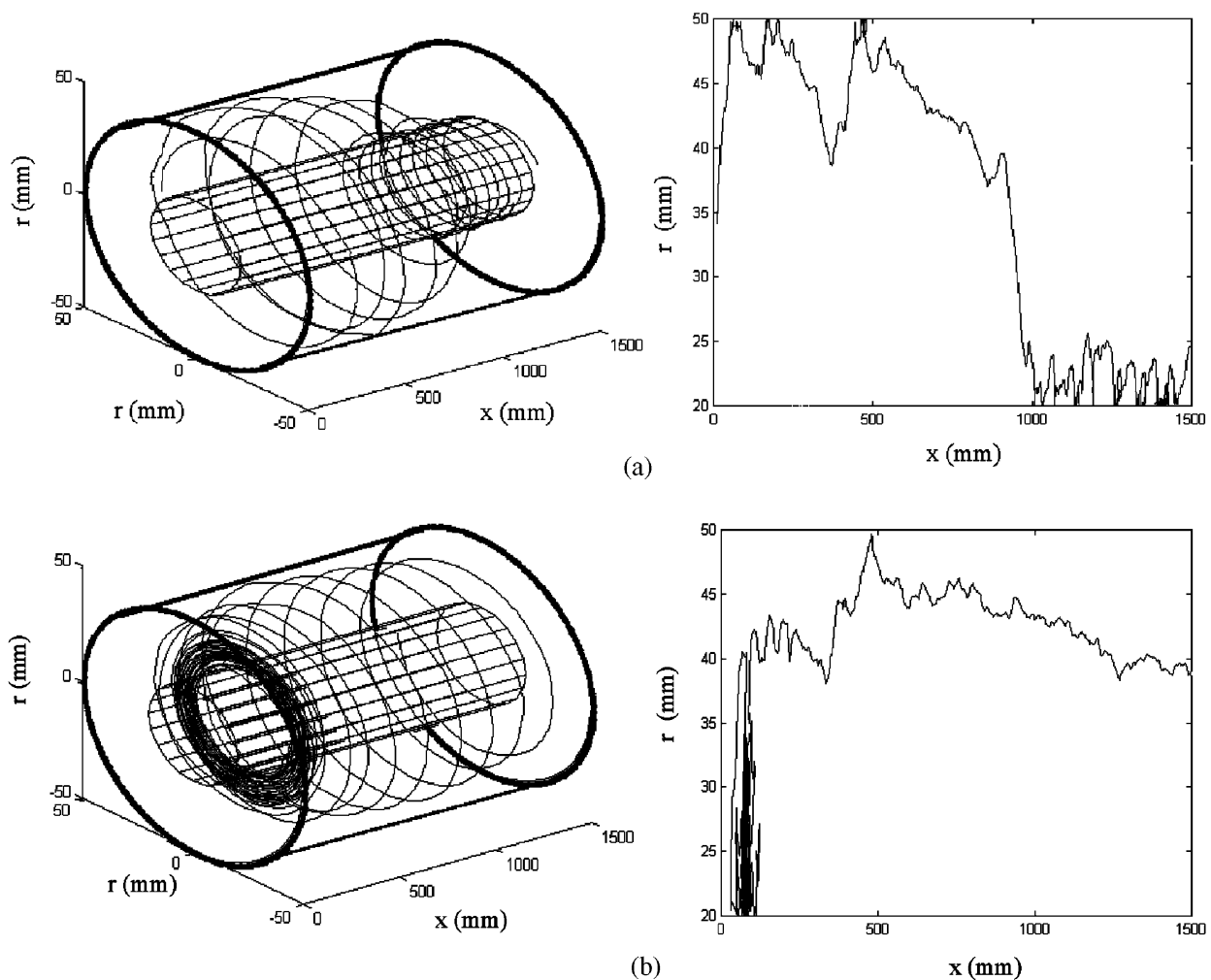

Fig. 10. Examples of calculated trajectories (three-dimensional representation and axial-radial plane projection): (a) first example; (b) second example.

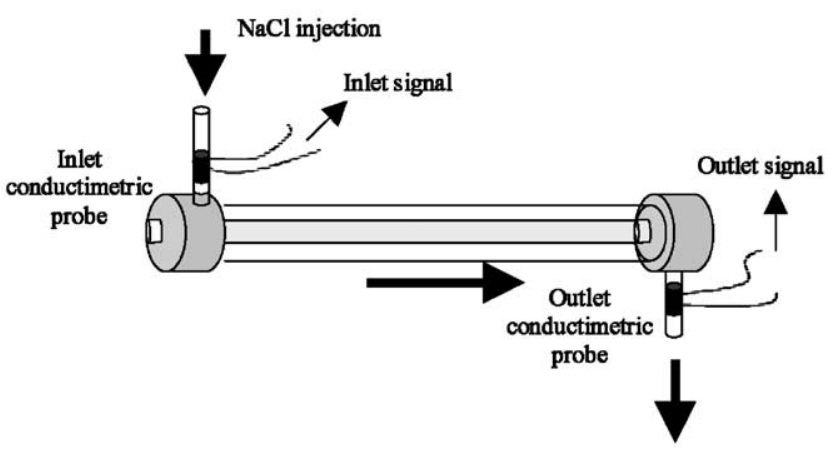

Fig. 11. Experimental setup for RTD determination.

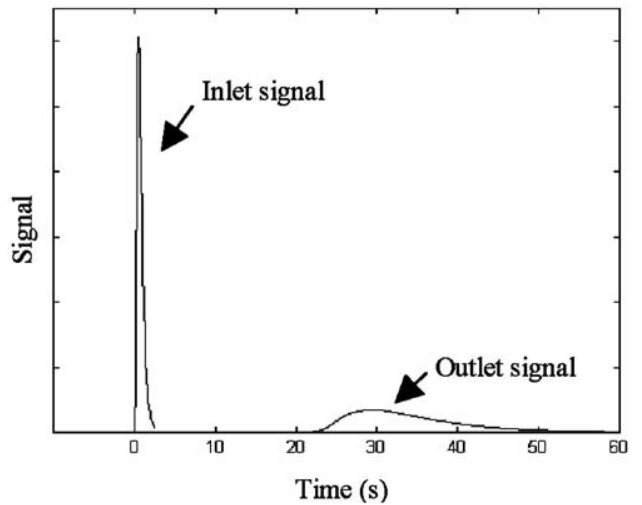

Fig. 12. Experimental results for RTD measurement.

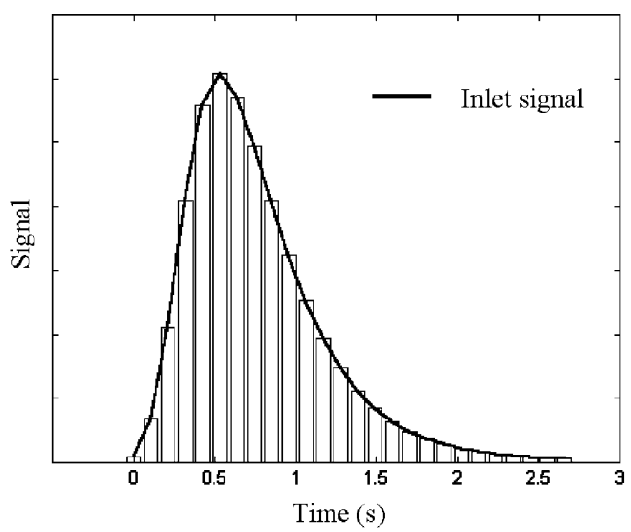

Fig. 13. Division of the inlet signal into time-step intervals

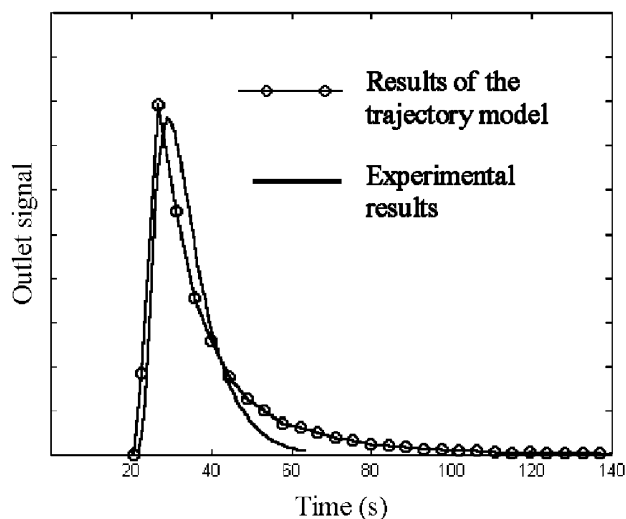

Fig. 14. Results of the trajectory calculation algorithm. 
to be an efficient means of enhancing transport phenomena in chemical reactors with a rather simple design. The trajectory model should lead to better control of a swirl reactor.

\section{Notation}

$c_{\text {cor }}$
$c_{\text {rand }}$
$e$
$f_{x, r, \xi}\left(l_{x}\right)$
$f_{x, r, \xi}\left(l_{r}\right)$
$g_{x, r, \xi}\left(l_{x}\right)$
$g_{x, r, \xi}\left(l_{r}\right)$
$K=T_{x}^{2}+T_{r}^{2}+T_{\xi}^{2}$
$L_{f x}$
$L_{f x}$
$L_{g r}$
$L_{f r}$
$L_{g x}$
$P$

$q(P)$

$q(m, \tau)$

$Q$

$r$

$R_{i}$

$\operatorname{Re}=2 e \bar{U} / v$

$R_{o}$

$T_{x}$

$T_{r}$

$T_{\xi}$

$\bar{U}=Q /\left(\pi\left(R_{o}^{2}-R_{i}^{2}\right)\right)$

$u$

$U$

$U^{\prime}$

$x$ weight linked to spatial correlation

weight linked to the random part of the fluctuating velocity

thickness of the annular gap, $m$ axial correlation of the axial velocity component

radial correlation of the axial velocity component

axial correlation of the radial velocity component

radial correlation of the radial velocity component dimensionless turbulent energy integral length scale, $\mathrm{m}$ integral length scale calculated for $f_{x, r, \xi}\left(l_{x}\right), \mathrm{m}$ integral length scale calculated for $g_{x, r, \xi}\left(l_{r}\right), \mathrm{m}$ integral length scale calculated for $f_{x, r, \xi}\left(l_{r}\right), \mathrm{m}$ integral length scale calculated for $g_{x, r, \xi}\left(l_{x}\right), \mathrm{m}$

position of the elementary fluid particle

Gaussian distributed random function for the location $P, \mathrm{~m} / \mathrm{s}$

Gaussian distributed random function of mean $m$ and root mean square $\tau$

flow rate in the annulus, $\mathrm{m}^{3} / \mathrm{s}$ radial position with respect to the annulus axis, $\mathrm{m}$

external radius of the inner cylinder, $\mathrm{m}$

Reynolds number [Eq. (1)]

internal radius of the outer cylinder, $\mathrm{m}$

axial turbulence intensity component

radial turbulence intensity component

circumferential turbulence intensity component

average velocity in the annulus, $\mathrm{m} / \mathrm{s}$ instantaneous velocity, $\mathrm{m} / \mathrm{s}$

mean velocity, $\mathrm{m} / \mathrm{s}$

fluctuating velocity, $\mathrm{m} / \mathrm{s}$

axial position with respect to the tangential inlet, $\mathrm{m}$

\section{Greek letters}

$\xi$

$v$

\section{Subscripts}

$x$

$r$

Other symbol

\langle\rangle intensity

\section{spatial averaging}

circumferential position with respect to the tangential inlet axis, rad kinematic viscosity of water, $\mathrm{m}^{2} / \mathrm{s}$

axial component of mean velocity, velocity fluctuation, instantaneous velocity or turbulence intensity radial component of mean velocity, velocity fluctuation, instantaneous velocity or turbulence intensity circumferential component of mean velocity, velocity fluctuation, instantaneous velocity or turbulence

\section{References}

Aouabed, H., Legentilhomme, P., \& Legrand, J. (1995). Wall visualization of swirling decaying flow using a dot-paint method. Experiments in Fluids, 19, 43-50.

Aouabed, H., Legentilhomme, P., Nouar, C., \& Legrand, J. (1994). Experimental comparison of electrochemical and dot-paint methods for the study of swirling flow. Journal of Applied Electrochemistry, 24, 619-625.

Berlemont, A., Chang, Z., \& Gouesbet, G. (1998). Particle Lagrangian tracking with hydrodynamic interactions and collisions. Flow, Turbulence and Combustion, 60, 1-18.

Berlemont, A., Desjonqueres, P., \& Gouesbet, G. (1990). Particle Lagrangian simulation in turbulent flows. International Journal of Multiphase Flow, 16(1), 19-34.

Chen, X. Q., \& Pereira, J. C. (1996). Computation of turbulent evaporating sprays with well-specified measurements: A sensitivity study on droplet properties. International Journal of Heat and Mass Transfer, 39(3), 441-454.

Chen, X. Q., \& Pereira, J. C. (1998). Computation of particle dispersion in turbulent liquid flows using an efficient Lagrangian trajectory model. International Journal for Numerical Methods in Fluids, 26, 345-364.

Domgin, J. F., Huilier, D., Burnage, H., \& Gardin, P. (1997). Coupling of a Lagrangian model with CFD code: Application to the numerical modelling of the turbulent dispersion of droplets in a turbulent pipe flow. Journal of Hydraulic Research, 35(4), 473-490.

Farias Neto, S. R., Legentilhomme, P., \& Legrand, J. (1998). Finite-element simulation of laminar swirling decaying flow induced by means of a tangential inlet in an annulus. Computational Methods in Applied Mechanics and Engineering, 165, 189-213.

Gupta, A., Lilley, D. G., \& Syred, N. (1984). Swirl Flow. Energy and Engineering Sciences Series. Tunbridge Wells, UK: Abacus Press.

Hinze, J. O. (1959). Turbulence. New York: McGraw-Hill.

Legentilhomme, P., Aouabed, H., \& Legrand, J. (1993). Developing mass transfer in swirling decaying flow induced by means of a tangential inlet. Chemical Engineering Journal, 52, 137-147.

Legentilhomme, P., \& Legrand, J. (1990). Overall mass transfer in swirling decaying flow in annular electrochemical cells. Journal of Applied Electrochemistry, 20, 216-222. 
Legentilhomme, P., \& Legrand, J. (1991). The effects of inlet conditions on mass transfer in annular swirling decaying flow. International Journal of Heat and Mass Transfer, 34, 1281-1291.

Legentilhomme, P., \& Legrand, J. (1995). Distribution des temps de séjour en écoulement tourbillonnaire annulaire induit par une entrée tangentielle du liquide. Canadian Journal of Chemical Engineering, $73,435-443$.

Legrand, J., Aouabed, H., Legentilhomme, P., Lefèbvre, G., \& Huet, F. (1997). Use of electrochemical sensors for the determination of wall turbulence characteristics in annular swirling decaying flows. Experimental Thermal and Fluid Science, 15, 125-126.
Ormancey, A., \& Martinon, J. (1984). Prediction of particle dispersion in turbulent flows. Physico-Chemical Hydrodynamics, 5(3/4), 229-244.

Pruvost, J., Legrand, J., Legentilhomme, P., \& Doubliez, L. (2000). Particle image velocimetry investigation of the flow-field of a 3D turbulent annular swirling decaying flow induced by means of a tangential inlet. Experiments in Fluids, 29(3), 291-301.

Pruvost, J., Legrand, J., \& Legentilhomme, P. (2001). Three-dimensional swirl flow velocity-field reconstruction using a neural network with radial basis functions. Journal of Fluids Engineering, 123(7), 920-927. 